\title{
Evaluation of the Seegene Anyplex STI-7 Detection technique (V1.1) in the Molecular Diagnosis of Chlamydia trachomatis and Neisseria Gonorrhoeae
}

Babacar Faye ${ }^{1,2,3^{*}}$, Hassane Rambou Rahma ${ }^{1}$, Salimata Gueye Diouf ${ }^{1}$, Mbacké Sembène ${ }^{4}$, Alioune Dièye ${ }^{3}$

${ }^{1}$ Laboratory of Molecular Biology, Military Hospital of Ouakam (HMO), Dakar, Senegal

${ }^{2}$ AIDS Program of the Senegalese Armed Forces, Senegal

${ }^{3}$ Service of Immunology, Department of Pharmacy, Faculty of Medicine, Pharmacy and Odontology of Cheikh Anta Diop University of Dakar, Senegal

${ }^{4}$ Genetics team of population management. Department of Animal Biology, Faculties of Science and Technology, Cheikh Anta Diop University of Dakar, Senegal

*Corresponding author: Dr. Babacar Faye

Abstract

Introduction: Sexually Transmitted Infections (STIs) remains a public health problem due to their high number in the world, the asymptomatic nature of several cases and the consequences on the fertility of untreated affected people. The objective was to assess the performance of the newly acquired Anyplex ${ }^{\text {TM }}$ STI-7 Detection (V1.1) technique compared to that used in routine Abbott Real Time m2000 in the detection of Chlamydia trachomatis and Neisseria gonorrhoeae. Materiel and Methods: Retrospective study on a population of 283 people who could potentially have an STI. All urine samples are run on both methods. AnyplexTM STI7 Detection (V1.1) capable of detecting 7 pathogens including Chlamydia trachomatis and Nesseria gonorrhoeae compared to the routine one that we will consider as the reference one: Abbott Real Time m2000 CT/ NG. Results: For Chlamydia trachomatis, the sensitivity is 100\% [50.01, 100], the specificity of $97.5 \%$ [95.39, 99.01], the positive predictive value of $40 \%$ [16.82, 68.73], the negative predictive value of $100 \%[98.61,100]$ and Cohen's Kappa of $0.56[0.45,0.66]$ and for Neisseria gonorrheae, the sensitivity amounts to $33.33 \%[6.14,79.23]$, the specificity to $99.28 \%$ [97.43, 99.80], the positive predictive value at 33.33\% [6.14, 79.23], the negative predictive value at $99.28 \%$ [97.43, 99.80] and Cohen's Kappa at 0.33 [0.20, 0.44]. Conclusion : Our results showed a good sensitivity for Chlamydiae trachomatis allowing a use of this test in first intention. An assessment on a larger number of positive samples to confirm or deny this trend.

Keywords. Evaluation, Anyplex ${ }^{\mathrm{TM}}$ STI-7 Detection (V1.1), Chlamydia trachomatis, Neisseria gonorrhoeae, Abbott $\mathrm{m} 2000 \mathrm{CT} / \mathrm{NG}$.

Copyright @ 2020: This is an open-access article distributed under the terms of the Creative Commons Attribution license which permits unrestricted use, distribution, and reproduction in any medium for non-commercial use (NonCommercial, or CC-BY-NC) provided the original author and source are credited

\section{INTRODUCTION}

Chlamydia trachomatis and Neisseria gonorrhoeae continue to be a public health problem. In 2016, there were 127 million new infections of Chlamydia trachomatis and 87 million of Neisseria gonorrhoeae [1]. In African regions, between 2009 and 2016, the estimated prevalence was between 6 and $8 \%$ for Chlamydia trachomatis in women, against 4 and 5\% for men; and between 0 and $2 \%$ for Neisseria gonorrhoeae in both sexes [1]. However, the WHO report published in 2015 shows that no STI surveillance program in the Africa region has been developed [2]. These infections are often asymptomatic [3]. They are likely to lead, particularly in women, to a high risk of sexual transmission of HIV (WHO, 2018) [1]. However, when they are symptomatic, they are manifested by cervicitis in women, epidydimitis in men or even urethritis [4]. However there are treatments against this kind of infections such as azithromicin or doxycycline for Chlamydia trachomatis [4, 5], however those against Neisseria gonorrhoeae meet resistance in particular to tetracycline, thus allowing the infection to continue at the same as that which will not have been subjected to any type of treatment [6]. Untreated infections have even more serious consequences on reproductive health such as pelvic inflammatory disease, ectopic pregnancy and infertility [4-7]. In Senegal, epidemiological data on syphilis and Hepatitis B in people infected with HIV are available, however other STIs are not mentioned [8]. In order for STI prevention and management to be effective, it is important to diagnose this kind of infection in time to avoid its consequences, but also to identify them precisely for effective treatment. Several 
techniques have been developed for a rapid and reliable diagnosis of these infections. Techniques such as microscopy are not very effective due to the intracellular characteristics of the two bacteria [5]; or the bacterial culture which is long and very expensive perhaps for Chlamydia trachomatis [5], but very effective for Neisseria gonorrhoeae in the context of the antibiotic susceptibility test [9]. There are also rapid detection tests based on immunological or immuno-enzymatic tests [10] such as the ELISA (enzymelinked immunosorbent assay) and RIA (radioimmunoassay) [11] tests. However, most of these direct diagnostic methods are not suitable for non-invasive samples such as urine and semen are less used due to their low sensitivity [5]. Molecular biology techniques have overcome these constraints, due to their sensitivity by amplification of DNA and RNA of bacteria, which can be visualized and quantified. The pathogen genome nucleotide sequence is unique and allows specificity in its molecular detection [11]. In addition to nucleic acid probes, some molecular biology techniques use polymerase chain reaction (PCR) amplification techniques [11]. Research into an even more reliable technique is necessary for better management of STIs.

The aim of our study is to evaluate the performance of the Anyplex ${ }^{\text {TM }}$ STI-7 Detection method (V1.1) compared to that usually used Abbott m2000 $\mathrm{CT} / \mathrm{NG}$ in the molecular diagnosis of $N$. gonorrhoeae and $C$. trachomatis.

\section{MATERIAL AND METHOD \\ Study Type and Population}

This is a retrospective study of a population of 283 people living with HIV, 184 men and 97 women. Patient first jet urine is collected in Abbott Cervi-Collect Specimen Collection Kit tubes (Abbott Molecular Inc., Des Plaines, Illinois, USA). The manipulations took place in the molecular biology laboratory of the Armed Forces AIDS program at the Ouakam Military Hospital (Senegal) between June 2018 and February 2019.

\section{Molecular Techniques}

Two diagnostic techniques based on the amplification of bacterial DNA were used: Abbott m2000 CT/NG (Abbott Molecular Inc., Des Plaines, Illinois, USA) and Anyplex ${ }^{\mathrm{TM}}$ STI-7 Detection (V 1.1) (Seegene, Korea, Seoul).

\section{Abbott m2000 CT/NG Molecular Technique}

The Abbott m2000 CT/NG method is based on PCR technology with homogeneous fluorescence detection in real time. The CT/NG test is intended for the double detection of Chlamydia trachomatis and Neisseria gonorrhoeae. For Neisseria gonorrhoeae, the target gene is the opa gene [12] while for Chlamydia trachomatis, m2000 targets two differents regions of the cryptic plasmid [13]. The DNA extraction from urine is carried out at the Abbott m2000sp machine using the
Abbott mSample Preparation System DNA kit according to the manufacturer's protocol. Amplification and detection are carried out using reagents from the Abbott real time CT/NG amplification reagent kit on m2000rt. The amplified CT/NG target sequences are detected using fluorescently labeled oligonucleotide probes [14].

\section{AnyplexTM STI-7 Detection Method (V 1.1) (Seegene, Korea, Seoul)}

Anyplex ${ }^{\text {TM }}$ STI-7 Detection (V 1.1) (Seegene, Korea, Seoul) is a qualitative in-vitro test using Multiplex PCR with real-time fluorescence detection capable of identifying 7 microorganisms including Chlamydia trachomatis, Neissseria gonorrhoea.

The DNA extraction is manual and was carried out using the ZYMO Quick DNA ${ }^{\mathrm{TM}}$ Miniprep Plus kit (ZYMO Reaserch Corp, CA, USA) from urine according to the manufacturer's protocol. The DNA concentration and purity are checked on the NanoDrop ${ }^{\mathrm{TM}}$ Lite spectrophotometer (ThermoFisher Scientific). Amplification and detection were carried out with the Anyplex ${ }^{\text {TM }}$ STI-7 Detection kit (V1.1) (Seegene, Korea, Seoul) in the Biorad CFX96 ${ }^{\mathrm{TM}}$ Real Time PCR System thermal cycler (Bio-Rad, Hercules, CA, USA). The Anyplex test is based on TOCE ${ }^{\text {TM }}$ technology (Tagging Oligonucleotide Cleavage and Extension) which allows the detection and identification of several genotypes of pathogens [15]. An internal control (CI), a positive and a negative controls are included to validate the process.

\section{STATISTICAL ANALYSIS}

Data collection was done on Epi-Info7. The evaluation of the Anyplex ${ }^{\mathrm{TM}}$ STI-7 Detection method (V 1.1) was made by the sensitivity and the specificity of the detection of Chlamydia trachomatis and Neisseria gonorrhoeae. Measuring positive predictive (PPV) and negative (NPV) values allowed the evaluation of the extrinsic values of the Anyplex ${ }^{\mathrm{TM}}$ STI-7 Detection (V 1.1). The Kappa coefficient allowed us to measure reproducibility. The calculations were made with Open Epi software, version 3.

\section{RESULTS \\ Chlamydia Trachomatis}

With the Seegene Anyplex ${ }^{\text {TM }}$ STI-7 Detection method (V1.1), 10 patients were diagnosed positive out of a population of 283 patients in total, i.e. a prevalence of $3.53 \%$ and 4 were positive by the Abbott m2000 Real Time CT/NG method (true positives) and 6 were detected negative (false positives) (Table 1a). The Abbott m2000 Real Time CT/NG method detected 4 out of 283 positive patients, i.e. a prevalence of $1.41 \%$, all 4 diagnosed positive by Seegene AnyplexTM STI-7 Detection (V1.1), therefore no false positives. So we have 273 real negatives. For the detection of $\mathrm{C}$. trachomatis, the Anyplex technique presented a sensitivity and specificity of $100 \%$ and $97.5 \%$ respectively. The positive and negative predictive values 
were $40 \%$ and $100 \%$. This technique is very sensitive. The value of Kappa is 0.56 , which shows an average agreement between the two methods (Table-2a).

\section{Neisseria Gonorrhoeae}

With the Seegene Anyplex ${ }^{\mathrm{TM}}$ STI-7 Detection method (V1.1), 3 patients were diagnosed positive out of a total population of 283 patients, i.e. a proportion of $1.06 \%$ and among these 3 positive patients, only 1 was positive by the method 2000 Abbott Real Time CT/NG (true positive), the other 2 being false positives; and for the Abbott m2000 Real Time CT/NG method, we also had 3 positives patients out of 283 , i.e. a prevalence of $1.06 \%$, only 1 was also diagnosed positive by the Seegene Anyplex ${ }^{\mathrm{TM}}$ STI-7 Detection method (V1.1), (true positive), the other 2 are therefore false negatives. For the detection of Neisseria gonorrhoeae, the Anyplex technique presented a sensitivity and a specificity of $33.33 \%$ and $99.28 \%$ respectively. The positive and negative predictive values were $33.33 \%$ and $99.28 \%$. The value of Kappa is 0.34 , which shows a weak agreement between the two methods (Table-2b).

Table-1: Crossed results of the two methods Abbott m2000 Real Time and Seegene Anyplex ${ }^{\mathrm{TM}}$ STI-7 Detection (V1.1)

1.a) Chlamydia trachomatis

\begin{tabular}{|l|l|l|l|l|}
\hline Seegene Anyplex & TMTI-7 Detection (V1.1) & \multicolumn{4}{|l|}{ Abbot Real Time m2000 } \\
\cline { 2 - 5 } & & Positive & Negative & Total \\
\cline { 2 - 5 } & Positive & 4 & 6 & 10 \\
\cline { 2 - 5 } & Negative & 0 & 273 & 273 \\
\cline { 2 - 5 } & Total & 4 & 279 & 283 \\
\hline
\end{tabular}

1.b) Neisseiria Gonorrhoeae

\begin{tabular}{|l|l|l|l|l|}
\hline \multirow{3}{*}{$\begin{array}{l}\text { Seegene } \\
\text { Anyplex }\end{array}$} & \multicolumn{4}{|l|}{ Abbot Real Time m2000 } \\
\cline { 2 - 5 } STI-7 Détection (V1.1) & & Positive & Negative & Total \\
\cline { 2 - 5 } & Positive & 1 & 2 & 3 \\
\cline { 2 - 5 } & Negative & 2 & 278 & 280 \\
\cline { 2 - 5 } & Total & 3 & 280 & 283 \\
\hline
\end{tabular}

Table-2a: Sensibility, specificity and predictive values Seegene Anyplex ${ }^{\text {TM }}$ STI-7 Detection method (V1.1) compared to Abbott m2000sp/rt (Chlamydia trachomatis)

\begin{tabular}{|l|l|l|l|l|l|}
\hline Methods & Sensibility \% (95\% IC) & $\begin{array}{l}\text { Specificity \% } \\
(\mathbf{9 5 \%} \text { IC) }\end{array}$ & $\begin{array}{l}\text { PPV \% } \\
(\mathbf{9 5 \%} \text { IC) }\end{array}$ & $\begin{array}{l}\text { NPV \% } \\
(\mathbf{9 5 \%} \text { IC) }\end{array}$ & $\begin{array}{l}\text { Kappa } \\
(\mathbf{9 5 \%} \text { IC) }\end{array}$ \\
\hline Anyplex Abbott & $\begin{array}{l}\text { (00\% } \\
{[50.01-100]}\end{array}$ & $\begin{array}{l}97.5 \% \\
{[95.39-99.01]}\end{array}$ & $40 \%$ & $100 \%$ & 0.56 \\
& {$[16.82-68.73]$} & {$[98.61-100]$} & {$[0.45-0.66]$} \\
\hline
\end{tabular}

Table-2b: Sensibility, specificity and predictive values Seegene Anyplex ${ }^{\text {TM }}$ STI-7 Detection method (V1.1) compared to Abbott m2000sp/rt (Neisseria gonorrhoeae)

\begin{tabular}{|l|l|l|l|l|l|}
\hline Methods & Sensibility \% (95\% IC) & Specificity \% (95\% IC) & $\begin{array}{l}\text { PPV \% } \\
(\mathbf{9 5 \%} \text { IC) }\end{array}$ & $\begin{array}{l}\text { NPV \% } \\
(\mathbf{9 5 \%} \text { IC) }\end{array}$ & $\begin{array}{l}\text { Kappa } \\
\text { (95\% IC) }\end{array}$ \\
\hline Anyplex Abbott & 33.33 & 99.28 & 33.33 & 99.28 & 0.34 \\
& {$[6.14-79.23]$} & {$[97.43-99.80]$} & {$[6.14-79.23]$} & {$[97.43-99.80]$} & {$[0.20-0.44]$} \\
\hline
\end{tabular}

Positive Predictive Value (PPV); Negative Predictive Value (NPV)

\section{DISCUSSION}

Concerning the frequency, in the case of Chlamydia trachomatis, it amounts to $3.53 \%$ for the Anyplex $^{\text {TM }}$ STI-7 Detection method (V1.1) against $1.41 \%$ for the Abbott m2000 Real Time CT/NG method which shows that this method is more sensitive than the reference one. Cohen's Kappa number was 0.563, that is, an average agreement between the two methods. This rate is lower than that of Berçot et al., (Kappa by 0.85), thus demonstrating good agreement in the detection of Chlamydia trachomatis [16].
For the case of Neisseria gonorrheae, concerning the frequency, it amounts to $1.06 \%$ for the Anyplex ${ }^{\mathrm{TM}}$ STI-7 Detection method (V1.1) as well as for the second method namely the Abbott m2000 Real Time CT/NG method which may let think that the two methods are equal. However, Cohen's Kappa which stands at 0.33 shows a weak agreement between the two methods. We can say here that the 2 methods are worth in agreement with the frequencies, however, the Kappa of Cohen indicates a weak agreement between these 2 methods. It is true that the frequencies are the same, however, they do not concern the same individuals as we see in the crosstab. Berçot et al, obtained a Kappa at 0.88 showing great agreement for their scenario [16]. 
Babacar Faye et al., Sch Acad J Biosci, Feb, 2020; 8(2): 46-50

The sensitivity for Chlamydia trachomatis is $100 \%$, which testifies to the very great efficiency of this method for the detection of a patient and such a value of the sensitivity is explained by the fact that there are no false negatives.

A sensitivity of $85.1 \%$ has been described by Berçot et al., who recommended it as a diagnostic method. Our result on sensitivity was found by Choe et al., who have higher specificity [17].

In the detection of Neisseria gonorrheae, the sensitivity is very low (33.33\%) which shows that this method is not recommended in this context. On the other hand, regarding the specificity, in the case of our study it is high, that is $99.23 \%$.

This low sensitivity suggests an increase in the number of positives for a better evaluation of this technique. This AnyplexII technique showed good sensitivity for Chlamydiae trachomatis and weak for Neisseria gonorrheae. The specificity and negative predictive value were good.

These results should be considered preliminary and an increase in the number of positives is necessary to confirm this trend.

However, this method can be proposed as the first screening for Chlamydiae trachomatis in a diagnostic algorithm. Studies on the comparison of these techniques are very rare which makes comparison difficult.

\section{CONCLUSION}

Our results showed a good sensitivity of Anyplex $^{\mathrm{TM}}$ STI-7 Detection method (V1.1) for Chlamydiae trachomatis allowing a use it in first intention. An assessment on a larger number of positive samples to confirm or deny this trend.

\section{ACKNOWLEDGMENTS}

The authors are grateful to the Department of Defense HIV/AIDS Prevention Program (DHAPP) for its support of molecular biology equipment and Africare for its collaboration. We would like to extend our acknowledgement to Pape Andre Diouf for his revision of the manuscript.

\section{REFERENCES}

1. Organisation Mondiale de la santé Report on global sexually transmitted infection surveillance, 2018, Genève, OMS, 2019. http://apps.who.int/iris/bitstream/hetle/10665/2772 58/9789241565691eng.pdf?ua=1

2. Organisation Mondiale de la santé, Report on global sexually transmitted infection surveillance 2015, Genève, OMS, 2016. disponible sur: http://apps.who.int/iris/bitstream/10665/249553/1/ 9789241565301eng.pdf?ua=1

3. Garcia M, Chessa C, Le Moal G, Aubry P, Pluzanski MF, Albouy-llaty M, Plouzeau C, Perraud E, Lévêque N, Beby-Defaux A. IST détectées au CeGIDD: prévalence et apport de la PCR multiplex. Médecine et Maladies Infectieuses. 2017 Jun 1;47(4):S1-2.

4. Yarbrough ML, Burnham CA. The ABCs of STIs: an update on sexually transmitted infections. Clinical chemistry. 2016 Jun 1;62(6):811-23.

5. Hamdad F, Orfila J. Diagnostic d'une infection uro-génitale à Chlamydia trachomatis. Apport des techniques d'amplification génique. Progrès en urologie. 2005;15:598-601.

6. Workowski KA, Bolan GA. Sexually transmitted diseases treatment guidelines, 2015. MMWR. Recommendations and reports: Morbidity and mortality weekly report. Recommendations and reports. 2015 Jun 5;64(RR-03):1.

7. Weström LA, Joesoef RI, Reynolds GL, Hagdu AL, Thompson SE. Pelvic inflammatory disease and fertility. A cohort study of 1,844 women with laparoscopically verified disease and 657 control women with normal laparoscopic results. Sexually transmitted diseases. 1992;19(4):185-92.

8. Centre National de Lutte contre le sida, enquête nationale de surveillance combinee des ist et du $\mathrm{vih} / \mathrm{sida} \quad$ (ensc), 2015. https://www.cnlssenegal.org/wp-content/uploads/2 018/06/RAPPORT-DE-SYNTHESEENSC-2015-1 .pdf

9. Verma R, Sood S. Gonorrhoea diagnostics: An update. Indian journal of medical microbiology. 2016 Apr 1;34(2):139.

10. Lavigne J, Jeandrot A, Sotto A. Les tests rapides de diagnostic des infections virales et parasitaires. Spectra biologie. 2006;151:34.

11. Madigan M. Brock biologie des micro-organismes. Paris: Pearson, 2007.

12. Tabrizi SN, Unemo M, Limnios AE, Hogan TR, Hjelmevoll SO, Garland SM, Tapsall J. Evaluation of six commercial nucleic acid amplification tests for detection of Neisseria gonorrhoeae and other Neisseria species. Journal of clinical microbiology. 2011 Oct 1;49(10):3610-3615.

13. Dize L, West S, Williams JA, Van Der Pol B, Quinn TC, Gaydos CA. Comparison of the Abbott m2000 RealTime CT assay and the Cepheid GeneXpert CT/NG assay to the Roche Amplicor CT assay for detection of Chlamydia trachomatis in ocular samples from Tanzania. Journal of clinical microbiology. 2013 May 1;51(5):1611-3.

14. Gaydos CA, Cartwright CP, Colaninno P, Welsch J, Holden J, Ho SY, Webb EM, Anderson C, Bertuzis R, Zhang L, Miller T. Performance of the Abbott RealTime CT/NG for detection of Chlamydia trachomatis and Neisseria gonorrhoeae. 
Babacar Faye et al., Sch Acad J Biosci, Feb, 2020; 8(2): 46-50

Journal of clinical microbiology. 2010 Sep 1;48(9):3236-43.

15. Boccoz SC. Développement de PCRs multiplexes pour le diagnostic: microarrays analytiques (Doctoral dissertation, Université Claude Bernard-Lyon I). 2016 Mar.

16. Berçot B, Amarsy R, Goubard A, Aparicio C, Loeung HU, Segouin C, Gueret D, Jacquier H, Meunier F, Mougari F, Cambau E. Assessment of coinfection of sexually transmitted pathogen microbes by use of the anyplex II STI-7 molecular kit. Journal of clinical microbiology. 2015 Mar 1;53(3):991-3.

17. Choe HS, Lee DS, Lee SJ, Hong SH, Park DC, Lee MK, Kim TH, Cho YH. Performance of Anyplex ${ }^{\mathrm{TM}}$ II multiplex real-time PCR for the diagnosis of seven sexually transmitted infections: comparison with currently available methods. International Journal of Infectious Diseases. 2013 Dec 1;17(12):e1134-40. 